\title{
ADIPONECTINA SÉRICA Y SU ASOCIACIÓN CON VARIABLES CARDIOMETABÓLICAS EN UNA POBLACIÓN ADULTA DE LA CIUDAD DE CARHUAMAYO (JUNÍN-PERÚ)
}

\author{
Serum adiponectin and its association with cardiometabolic variables in an adult population \\ from Carhuamayo (Junín-Perú)
}

Elizabeth Carranza ${ }^{1}$, Haydée Zúñiga², Carmen Peña², Miguel Huarcaya², Gustavo Guerra², Gloria Gordillo², Elizabeth Gonzáles $^{2}$ y Alejandro Florentini ${ }^{3}$

${ }^{1}$ Instituto Nacional de Biología Andina, Facultad de Medicina. ${ }^{2}$ Facultad de Farmacia y Bioquímica. ${ }^{3}$ Facultad de Ciencias Biológicas. Universidad Nacional Mayor de San Marcos.

\section{RESUMEN}

El objetivo del presente estudio fue determinar la asociación entre niveles séricos de adiponectina y factores de riesgo cardiometabólico en una población que habita en lugares de grandes alturas. Se incluyeron 88 personas adultas ( 31 hombres y 57 mujeres), nativas de la ciudad de Carhuamayo, $4146 \mathrm{msnm}$. Se registraron índices antropométricos y presión arterial; se determinaron los niveles séricos del perfil lipídico, glucosa y adiponectina. Se encontró que los valores medios de adiponectina sérica en mujeres eran significativamente más altos que en varones. La prevalencia de hipoadiponectinemia $(<4 \mu \mathrm{g} / \mathrm{mL})$ fue de $23,9 \%$ en la población total, $45,2 \%$ en hombres y 12,4\% en mujeres; la mayor prevalencia de síndrome metabólico (47,6 \%) correspondió al grupo hipoadiponectinémico. El nivel de adiponectina sérica presentó correlación: negativa con IMC y las concentraciones séricas de triglicéridos, y positiva con las concentraciones séricas de HDL-colesterol; ambas diferencias fueron estadísticamente significativas. Se concluye que, la hipoadiponectinemia está fuertemente asociada con el síndrome metabólico y la hipertrigliceridemia, que es el componente prevalente del síndrome metabólico en esta población.

Palabras clave: Adiponectina, perfil lipídico, síndrome metabólico, altitud.

\section{SUMMARY}

The objective of this study was to determine the association between serum adiponectin levels and cardiometabolic risk factors in a high altitude population. We included 88 adults (31 men and 57 women), natives from Carhuamayo, town at $4146 \mathrm{~m}$. Anthropometric data were recorded and blood pressure was measured. Serum levels of lipidic profile, glucose and adiponectin were determined. Mean values of serum adiponectin in women were found to be significantly higher than those in men. Estimated hipoadiponectinemia $(<4 \mu \mathrm{g} / \mathrm{mL})$ was $23,9 \%$ in the total sample, $45,2 \%$ in men and $12,4 \%$ in women; the serum adiponectin highest prevalence $(47,6 \%)$ corresponded to the hipoadiponectinemic group. Serum adiponectin level showed significantly negative correlation with BMI and with serum triglyceride concentration, and was positively and significantly correlated with HDL-cholesterol. Was concluded that hipoadiponectinemia is highly associated with metabolic syndrome and with hypertriglyceridemia, which is the most prevalent component of metabolic syndrome in the population studied.

Keywords: Adiponectin, lipidic profile, metabolic syndrome, high altitude.

\section{INTRODUCCIÓN}

a adiponectina es una de las más abundantes adipocinas secretadas por los adipocitos. Es una proteína de $30 \mathrm{kDa}$ de masa molecular, compuesta por 244 aminoácidos, y cuya estructura contiene un dominio $\mathrm{N}$-terminal semejante al colágeno y un dominio C-terminal globular semejante al factor de complemento $\mathrm{C}_{1 \mathrm{q}}{ }^{(1)}$. Su secreción es estimulada por la insulina, el factor de crecimiento similar a la insulina (IGF-1, insulin growth factor 1), los receptores activadores de la proliferación peroxisomal (PPARg, peroxisome proliferator activated nuclear receptor $\gamma$ ) y el LRH-1 (liver receptor homolog-1); los dos últimos implicados en la regulación de la síntesis de adiponectina por activación de la transcripción del gen de adiponectina ${ }^{(2)}$.

La concentración de adiponectina circulante en el plasma representa aproximadamente el o,o1 \% del total de las proteínas plasmáticas. En contraste con otras adipocinas, cuyos niveles plasmáticos aumentan con incremento del tejido adiposo, los niveles plasmáticos de adiponectina disminuyen en individuos obesos ${ }^{(3)}$.

Laadiponectinaejercediferentesfuncionesreguladorasen el organismo, es un potente modulador del metabolismo de la glucosa y de los lípidos, aumenta la sensibilidad 
a la insulina, presenta acciones antiaterogénicas, antidiabéticas y antiinflamatorias. Se ha observado en diferentes estudios transversales en humanos que, los niveles de adiponectina plasmática correlacionan negativamente con la obesidad ${ }^{(3)}$, la resistencia a la insulina ${ }^{(4)}$, el nivel plasmático de triglicéridos ${ }^{(5)}$, la diabetes ${ }^{(6)}$ y las enfermedades cardiovasculares ${ }^{(7,8)}$. Una disminución del nivel de adiponectina plasmática (hipoadiponectinemia) se asocia al síndrome metabólico (SM) ${ }^{(5)}$. Se ha encontrado también que los niveles de adiponectina plasmática están influenciados por el sexo, la edad, grupo étnico y estilo de vida ${ }^{(9)}$.

Estudios fisiológicos, clínicos y epidemiológicos demuestran que hay menor incidencia de enfermedades coronarias en los residentes de altura que en los de nivel del mar ${ }^{(10)}$. De los factores de riesgo implicados, se conoce que en los habitantes de las grandes alturas (GA), la tasa de colesterol es menor y los niveles de HDL-colesterol (HDL-col) son mayores que a nivel del mar; se sabe también que es menor la prevalencia de hipertensión arterial y que el diabético de altura está en menor riesgo de presentar enfermedad cerebrovascular que el diabético de nivel del mar, en razón de las menores alteraciones lipídicas que presenta ${ }^{(10)}$. Sin embargo, no se conocen los valores de adiponectina sérica en poblaciones que habitan en las grandes alturas, ni su relación con factores de riesgo cardiometabólicos.

Por lo expuesto, el propósito de este estudio fue evaluar el nivel de adiponectina sérica, su distribución y relación con varios factores de riesgo cardiovascular y la prevalencia de SM en una población aparentemente sana de la ciudad de Carhuamayo a $4160 \mathrm{~m}$ de altitud.

\section{MATERIALES Y MÉTODOS}

\section{Sujetos de experiencia}

El presente es un estudio transversal en el que se incluyeron a 88 personas adultas, 31 hombres y 57 mujeres, con edades promedio de 44,1 y 44,2 años, respectivamente, nativas de la ciudad de Carhuamayo (Región Junín, 4146 msnm). Ninguno de ellos padecía de diabetes mellitus ni enfermedad cardiovascular.

Los sujetos de estudio se presentaron a primera hora de la mañana para la toma de muestra respectiva, en condiciones de ayuno absoluto (12 horas previas) y sin haber realizado actividad física.

\section{Recolección de las muestras}

Las muestras sanguíneas, $8 \mathrm{~mL}$, fueron obtenidas mediante punción venosa del antebrazo previa asepsia, y recibidas en tubos de ensayo sin anticoagulante, limpios y esterilizados. La sangre extraída se dejó reposar por 30 minutos para la coagulación y luego se centrifugó por 5 minutos a 4000 rpm para separar el suero. Se colocaron los sueros entre 2 y $5^{\circ} \mathrm{C}$ en un recipiente hermético, para ser transportados al laboratorio del Instituto Nacional de Biología Andina, y almacenados $\mathrm{a}-40^{\circ} \mathrm{C}$ hasta el momento del análisis.

\section{Mediciones}

La medición de la presión arterial la efectuó un profesional de la salud en el brazo izquierdo, con la persona sentada; se pesaron y tallaron los individuos sin calzado y con ropa ligera; asimismo, se midieron los perímetros de cintura y cadera.

\section{Determinaciones}

Se determinaron: la concentración de hemoglobina $(\mathrm{Hb})$ en sangre total y los niveles séricos de glucosa, colesterol total, HDL-col y triglicéridos por métodos enzimático-colorimétricos convencionales usando kits comerciales. La concentración de LDLcolesterol (LDL-col) se determinó aplicando la fórmula de Friedewald ${ }^{(11)}$. La adiponectina se determinó por el método de ELISA, utilizando un kit comercial marca Phoenix Pharmaceutical, INC. (USA).

De acuerdo a normas internacionales, el índice de masa corporal (IMC, peso en $\mathrm{kg}$, dividido entre el cuadrado de la estatura, en metros) entre $25 \leq \mathrm{IMC}<30$ $\mathrm{kg} / \mathrm{m}^{2}$ fue considerado como criterio de sobrepeso y el IMC $>30 \mathrm{~kg} / \mathrm{m}^{2}$, como criterio de obesidad ${ }^{(11)}$.

El SM se definió, de acuerdo a los criterios del National Cholesterol Education Program (NCEP) Adult Treatment Panel III (ATP III) ${ }^{(11)}$. Se consideró $\mathrm{SM}$ a la presencia de tres o más de los siguientes criterios: la presión sistólica y diastólica $\geq 135 / 85$ $\mathrm{mmHg}$, circunferencia de la cintura $>102 \mathrm{~cm}$ para hombres y $>88 \mathrm{~cm}$ para las mujeres, glucosa en ayuno $\geq 100 \mathrm{mg} / \mathrm{dL}$, HDL-col $\leq 40 \mathrm{mg} / \mathrm{dL}$ en hombres $\mathrm{y} \leq 50$ $\mathrm{mg} / \mathrm{dL}$ en mujeres y triglicéridos $\geq 150 \mathrm{mg} / \mathrm{dL}$.

Las personas fueron separadas en tres grupos de acuerdo a los niveles séricos de adiponectina, tal como ha sido propuesto anteriormente (7): <4,0 $\mu \mathrm{g} /$ $\mathrm{mL} ; 4,0-7,0 \mu \mathrm{g} / \mathrm{mL}$ y $>7,0 \mu \mathrm{g} / \mathrm{mL}$. Se define como hipoadiponectinemia a una concentración sérica de adiponectina $<4,0 \mu \mathrm{g} / \mathrm{mL}$ para ambos sexos.

\section{Análisis estadístico}

Los resultados fueron expresados en valores medios y desviación estándar. Se compararon las medias según la prueba $t$ de Student para dos muestras, y mediante el análisis de varianza para más 
Tabla 1. Características de la población de estudio según género.

\begin{tabular}{|c|c|c|c|}
\hline & $\begin{array}{c}\text { Hombres } \\
\text { (Media } \pm \text { DE) }\end{array}$ & $\begin{array}{c}\text { Mujeres } \\
\text { (Media } \pm \text { DE) }\end{array}$ & $p$ \\
\hline $\mathrm{n}$ & 31 & 57 & \\
\hline Edad, años & $44,1 \pm 13,0$ & $44,2 \pm 13,6$ & 0,966 \\
\hline Peso, kg & $67,5 \pm 8,7$ & $59,9 \pm 9,4$ & $0,000^{*}$ \\
\hline Talla, m & $1,7 \pm 0,1$ & $1,5 \pm 0,1$ & $0,000^{*}$ \\
\hline IMC, $\mathrm{kg} / \mathrm{m}^{2}$ & $24,1 \pm 2,6$ & $25,3 \pm 3,9$ & 0,072 \\
\hline $\begin{array}{l}\text { Cintura, cm } \\
\text { Cadera, cm }\end{array}$ & $\begin{array}{l}92,7 \pm 7,0 \\
98,2 \pm 5,0\end{array}$ & $\begin{array}{l}92,5 \pm 10,4 \\
101,4 \pm 9,3\end{array}$ & $\begin{array}{l}0,886 \\
0,037^{*}\end{array}$ \\
\hline $\begin{array}{l}\text { Presión sistólica, mmHg } \\
\text { Presión diastólica, mmHg }\end{array}$ & $\begin{array}{l}12,5 \pm 1,7 \\
7,7 \pm 1,2\end{array}$ & $\begin{array}{l}11,8 \pm 1,5 \\
7,7 \pm 1,3\end{array}$ & $\begin{array}{l}0,064 \\
0,966\end{array}$ \\
\hline $\begin{array}{l}\text { Sobrepeso }(\%)^{\mathrm{a}} \\
\text { Obesos }(\%)^{\mathrm{a}} \\
\text { Síndrome metabólico }(\%)^{\mathrm{b}}\end{array}$ & $\begin{array}{l}35,5 \\
3,2 \\
16,1\end{array}$ & $\begin{array}{l}33,3 \\
15,8 \\
31,6\end{array}$ & $\begin{array}{l}0,839 \\
0,075 \\
0,038^{*}\end{array}$ \\
\hline
\end{tabular}

En la comparación de medias de variables continuas se aplicó la prueba $t$ de Student

* Estadísticamente significativo

a Prueba z para proporciones

${ }^{b} \boldsymbol{X}^{2}$ para porcentajes

de dos muestras. Se compararon los porcentajes según la prueba chi-cuadrado, o bien según la prueba z. Se evaluó el grado de asociación mediante el coeficiente de correlación de Pearson. Se consideró significativo todo resultado cuyo valor asociado de $p$ fuera $<0,05$.

\section{RESULTADOS}

La tabla 1 muestra las características de la población participante. La media de edad de los 31 hombres fue de 44,1 \pm 13 ,o años y de las 57 mujeres fue 44,2 \pm 13.6 años, sin diferencia significativa $(p=0,966)$. No se encontraron diferencias significativas por sexo en IMC, cintura, presión sistólica y presión diastólica. Los porcentajes de sobrepeso y de obesos de la muestra total fueron $34,1 \%(33,3 \%$ en mujeres y $35,5 \%$ en hombres) y $11,4 \%$ (3,2\% en hombres y $15,8 \%$ en mujeres), respectivamente.

El 50\% de la población total presenta, en $\mathrm{cm}$, valores de cintura mayor a lo establecido, con alta significación estadística entre hombres y mujeres (9,7\% y $71,9 \%$, respectivamente). En relación a la cadera, los valores, en $\mathrm{cm}$, que presentan las mujeres son mayores que los de hombres: 101,4 \pm 9,3 y $98,2 \pm 5, \mathrm{o}(p=0,037)$.

La prevalencia de SM en la muestra total analizada fue de $26,1 \%$; los hombres presentaron una prevalencia de $16,1 \%$ y las mujeres de $31,6 \%$, con diferencias significativas por género $(p=0,038)$.

Los resultados de hemoglobina y demás parámetros bioquímicos se presentan en la tabla 2. Los niveles de hemoglobina en hombres $(18,6$ $\pm 1,9 \mathrm{~g} / \mathrm{dL})$ y en mujeres $(17,2 \pm 2, \mathrm{og} / \mathrm{dL})$ difieren estadísticamente, $(p=0,002)$. No se encontraron diferencias significativas por sexo en cuanto a niveles séricos de glucosa y parámetros lipídicos. Sin embargo, el análisis de frecuencias $\left(x^{2}\right)$ encontró que $13,6 \%$ de la población presentaba valores de glucosa por encima de $100 \mathrm{mg} / \mathrm{dL}$, de los cuales $19,4 \%$ corresponde a varones y $10,5 \%$ a mujeres, sin significación estadística. El $22,7 \%$ de los participantes presentaban niveles de hipercolesterolemia (colesterol $\geq 200 \mathrm{mg}$ / $\mathrm{dL}$ ), aunque sin diferencia significativa entre hombres, $16,1 \%$, y mujeres, $26,3 \%,(p=0,276)$. $\mathrm{El} 42 \%$ de la población total presentó valores bajos de HDL-col $(<40 \mathrm{mg} / \mathrm{dL})$, sin diferencia significativa entre hombres $48,4 \%$, y mujeres 63,2\%, $(p=0,180)$; y 55,7\% presenta niveles de hipertrigliceridemia (triglicéridos $\geq 150 \mathrm{mg} / \mathrm{dL}$ ), sin diferencia significativa entre hombres $61,3 \%$ y mujeres $52,6 \%,(p=0,435)$.

La comparación de los valores medios de adiponectina: 5,3 \pm 4,0 $\mu \mathrm{g} / \mathrm{mL}$ (Intervalo de Confianza, CI del 95\% de 3,7 a 6,7 $\mu \mathrm{g} / \mathrm{mL}$ ) para hombres, y 9,0 \pm 4,6 $\mu \mathrm{g} / \mathrm{mL}$ (CI del 95\% de 7,8 a 10,2 $\mu \mathrm{g} / \mathrm{mL}$ ) para mujeres, dio como resultado una diferencia significativa entre ambos sexos, $(p=o, o o o)$. Se encontró prevalencia de hipoadiponectidemia del $23,9 \%$ en la población total; en hombres $45,2 \%$ y en mujeres $12,3 \%$. El grupo considerado como límite normal inferior presentó una prevalencia del $27,3 \%$ y el grupo considerado normal una prevalencia de 48,9\% (figura 1).

Se evaluó el grado de asociación entre el nivel de adiponectina sérica y las demás variables observadas (tabla 3). Se encontró una estrecha relación directa entre niveles séricos de adiponectina y los de HDLcol $(p=0,00 o)$; y relación inversa entre adiponectina e IMC $(p=0,005)$, triglicéridos $(p=0,022)$ y hemoglobina $(p=0,005)$. No se encontró asociación significativa entre el nivel de adiponectina sérica con la edad y los parámetros evaluados. Cuando la relación encontrada se ajustó por
Tabla 2. Variables bioquímicas de la población de estudio.

\begin{tabular}{lccc}
\hline & $\begin{array}{c}\text { Hombres } \\
\text { (Media } \pm \text { DE) }\end{array}$ & $\begin{array}{c}\text { Mujeres } \\
\text { (Media } \pm \text { DE) }\end{array}$ & $p$ \\
\hline $\mathrm{n}$ & 31 & 57 & \\
Hemoglobina, g/dL & $18,6 \pm 1,9$ & $17,2 \pm 2,0$ & $0,002^{*}$ \\
Glucosa, mg/dL & $85,1 \pm 16,7$ & $88,1 \pm 11,1$ & 0,315 \\
Colesterol, mg/dL & $168,3 \pm 37,5$ & $176,6 \pm 31,2$ & 0,298 \\
HDL-colesterol, mg/dL & $41,6 \pm 10,5$ & $45,5 \pm 10,3$ & 0,102 \\
LDL-colesterol, mg/dL & $89,1 \pm 34,1$ & $93,8 \pm 28,6$ & 0,521 \\
Triglicéridos, mg/dL & $187,9 \pm 89,3$ & $186,8 \pm 91,0$ & 0,954 \\
Adiponectina, $\mu \mathrm{gg} / \mathrm{mL}$ & $5,3 \pm 4,0$ & $9,0 \pm 4,6$ & $0,000^{*}$ \\
\hline
\end{tabular}

${ }^{*}$ Estadísticamente significativo (t de Student para medias) 
la variable de confusión sexo, se mantuvo la relación entre adiponectina, IMC, HDL-col y triglicéridos, en tanto que con la hemoglobina no alcanzó significación estadística (figura 2).

La figura 3 muestra la prevalencia de SM según el nivel de adiponectina sérica. La prevalencia de SM fue de 47,6; 16,7 y 20,9\% en los grupos de $<4 \mu \mathrm{g} / \mathrm{mL}, 4-7 \mu \mathrm{g} / \mathrm{mLy}>7 \mu \mathrm{g} / \mathrm{mL}$, respectivamente. Se observa fuerte asociación entre hipoadiponectinemia $(<4 \mu \mathrm{g} / \mathrm{mL})$ y $\mathrm{SM}$, $(p=0,037)$.

Los valores medios de adiponectina sérica $(\mu \mathrm{g} / \mathrm{mL})$, según número de componentes de $\mathrm{SM}$, se presentan en la tabla 4. Aunque no se encontró diferencia significativa entre las medias de adiponectina, se observa una tendencia a disminuir sus valores desde la presencia de un componente, de tal manera que el valor medio

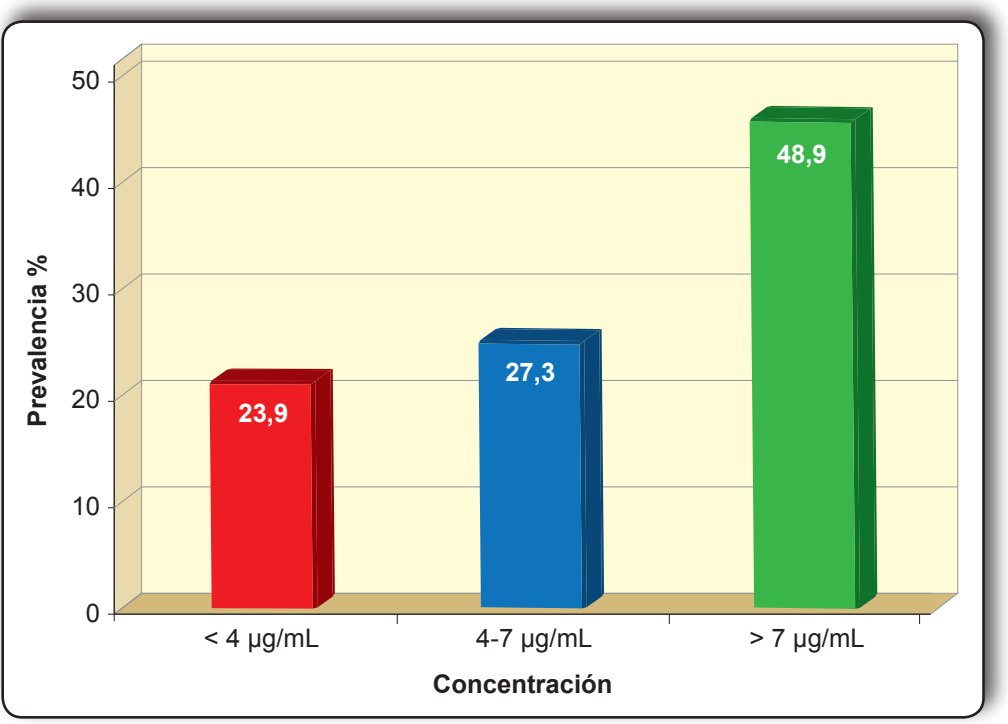

Figura 1. Distribución de la población según niveles séricos de adiponectina. de adiponectina estimado por regresión lineal sobre el número de componentes, disminuye a razón de 1,019 $\mu \mathrm{g} /$ $\mathrm{mL}(p=0,023)$ por cada unidad adicional de componente.

Con el fin de comparar la prevalencia estimada de cada componente del SM en la población de estudio, la muestra se dividió en dos grupos de acuerdo a la concentración de adiponectina: $<4 \mu \mathrm{g} / \mathrm{mL}$ (hipoadiponectinemia) $\mathrm{y} \geq 4 \mu \mathrm{g} / \mathrm{mL}$ (figura 4 ).

Los sujetos con hipoadiponectinemia, $<4,0 \mu \mathrm{g} / \mathrm{mL}$, mostraron, respecto a los que tenían una concentración $\geq 4,0 \mu \mathrm{g} / \mathrm{mL}$ : menor prevalencia de obesidad abdominal, $(49,2 \%$ vs $52,2 \% ; p=0,617)$; mayor frecuencia de hipertensión, (38,1\% frente a 23,0\%; $p=0,320)$; niveles

Tabla 3. Correlación entre nivel de adiponectina y variables de riesgo cardiometabólico.

\begin{tabular}{lcc}
\hline \multicolumn{1}{c}{ Variable } & $\boldsymbol{r}$ & $\boldsymbol{p}$ \\
\hline Edad, años & 0,143 & 0,183 \\
Peso, kg & $-0,481$ & $0,000^{*}$ \\
Talla, m & $-0,291$ & $0,006^{*}$ \\
IMC, kg/m2 & $-0,294$ & $0,005^{*}$ \\
Cintura, cm & $-0,187$ & 0,081 \\
Cadera, cm & $-0,161$ & 0,134 \\
Presión sistólica, mmHg & $-0,100$ & 0,354 \\
Presión diastólica, mmHg & 0,087 & 0,420 \\
Glucosa, mg/dL & 0,138 & 0,205 \\
Colesterol, mg/dL & $-0,047$ & 0,663 \\
HDL-colesterol, mg/dL & 0,394 & $0,000^{*}$ \\
LDL-colesterol, mg/dL & $-0,043$ & 0,689 \\
Triglicéridos, mg/dL & $-0,244$ & $0,022^{*}$ \\
Hemoglobina (g/dL) & $-0,295$ & $0,005^{*}$ \\
\hline * Estadisticamentesignifcativo (r Pearson &
\end{tabular}

* Estadísticamente significativo ( $r$ de Pearson) altos deglucosa en ayunas (19,0\% frente a $11,9 \% ; p=0,643)$ y HDL-col disminuido ( $76,2 \%$ vs $59,7 \% ; p=0,267$ ), aunque sin significación estadística. La prevalencia de hipertrigliceridemia $(81,0 \%$ vs $47,8 \% ; p=0,016)$ y SM (47,96\% vs $19,4 \% ; p=0,022)$ fueron sigficativamente mayores en los sujetos con hipoadiponectinemia.

\section{DISCUSIÓN}

Se realizó un estudio transversal en una muestra de sujetos oriundos de las grandes alturas (Carhuamayo, $4161 \mathrm{msnm}$ ) para determinar la relación entre los niveles de adiponectina sérica y algunas variables consideradas como factores de riesgo de enfermedades cardiometabólicas. Así, se encontró que las mujeres en promedio presentaron mayor circunferencia abdominal; en cambio el IMC, la presión arterial y la circunferencia de cintura, en hombres están dentro de los parámetros establecidos como normales (11). En relación a la prevalencia del SM, se encontró que en la población total y por género, era mayor que lo reportado por otros autores ${ }^{(12)}$.

Tabla 4. Valores medios de adiponectina sérica $(\mu \mathrm{g} / \mathrm{mL})$ y número de componentes de síndrome metabólico.

\begin{tabular}{cccc}
\hline $\begin{array}{c}\text { Número de } \\
\text { componentes }\end{array}$ & n & Media & DE \\
\hline 0 & 7 & 9,3 & 5,9 \\
1 & 20 & 9,7 & 6,2 \\
2 & 29 & 7,1 & 3,9 \\
3 & 22 & 6,9 & 3,6 \\
4 & 10 & 6,1 & 3,9 \\
\hline
\end{tabular}

Ecuación de regresión lineal:

Adiponectina $=9,844-1,019^{*} \mathrm{~N}^{\circ}$ componentes $(p=o, o 23)$ 

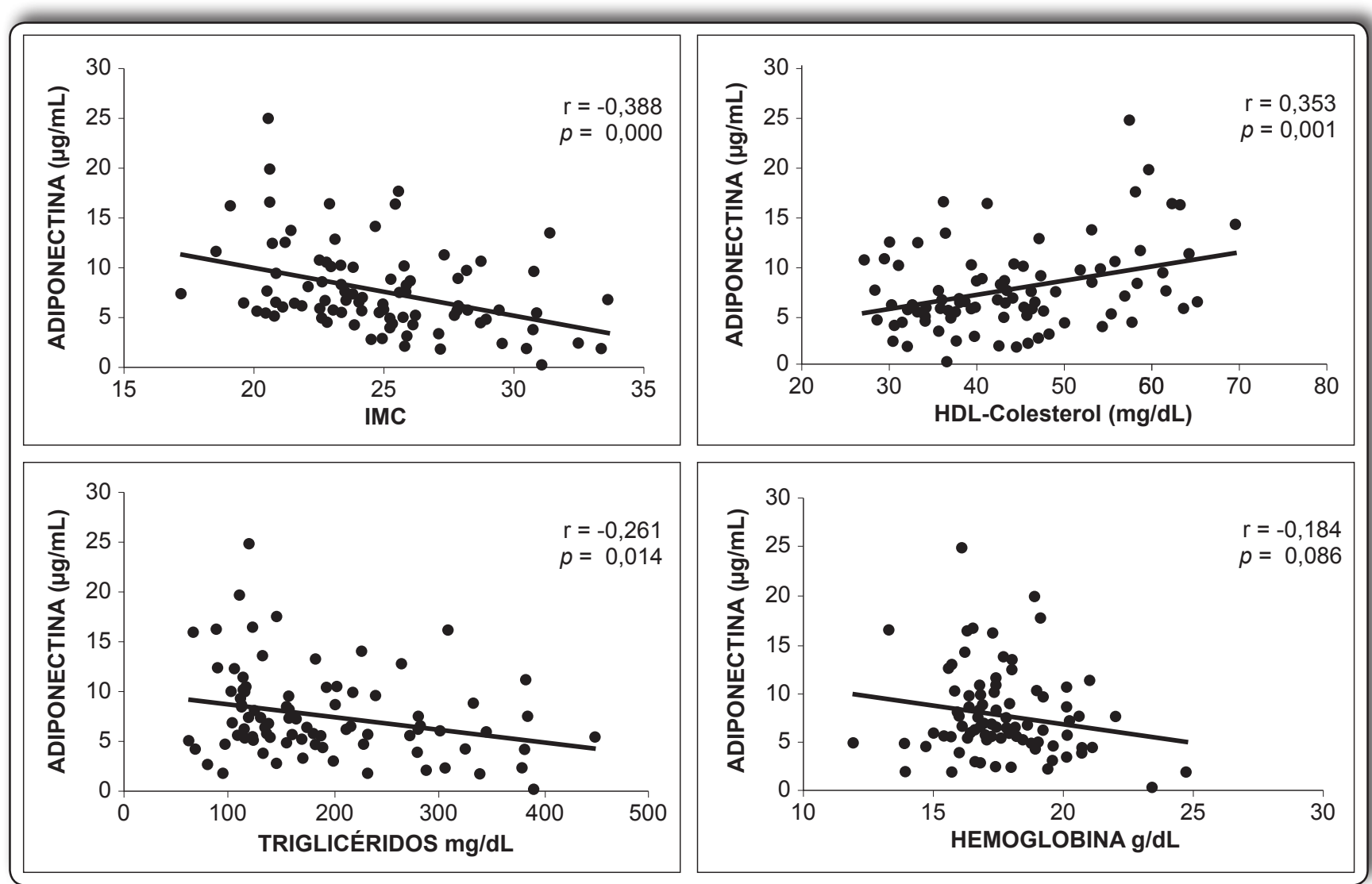

Figura 2. Relación entre adiponectina ajustada para sexo con IMC, HDL-col, triglicéridos y hemoglobina.

Los niveles de glucemia fueron similares en varones y mujeres y estaban dentro de los valores establecidos como normales. Los resultados de perfil lipídico, obtenidos en este estudio, indican que los valores séricos de colesterol total y LDL-col estaban dentro del rango considerado deseable según la literatura ${ }^{(11)}$ y eran similares a los encontrados por otros investigadores ${ }^{(12,13)}$. Aunque los niveles séricos de HDL-col aquí observados dentro de límites considerados como "riesgo moderado" difieren de los referidos por otros autores ${ }^{(12,13)}$, quienes encontraron valores altos en otras poblaciones andinas, dichos niveles son muy parecidos a los reportados por Gonzales y Tapia ${ }^{(10)}$, quienes también trabajaron en Carhuamayo. El porcentaje de hipertrigliceridemia fue alto, similar al de diversos trabajos en otras poblaciones que viven en las grandes alturas; se cree que la causa sería el alto contenido de carbohidratos en la dieta que consumen las poblaciones del Ande ${ }^{(10,12,13)}$.

Los resultados del presente trabajo, muestran que los valores medios de adiponectina en mujeres son significativamente más altos $(p=o, o o o)$ que en los varones; estos resultados concuerdan con los obtenidos por otros autores ${ }^{(14-16)}$; aparentemente esta diferencia se inicia después de la pubertad, pues se ha demostrado disminución progresiva de los niveles de adiponectina, en paralelo con el desarrollo físico y la pubertad en los niños en comparación con las niñas; este descenso está fuertemente asociado a los niveles de andrógenos y puede explicar las diferencias de género en adultos ${ }^{(15)}$. Im JA y col. confirmaron que los estrógenos ejercerían un efecto estimulatorio, mientras que los andrógenos causan supresión de la producción de adiponectina ${ }^{(16)}$

Diversas publicaciones $(7,9,17)$ dan cuenta que niveles de adiponectina sérica por debajo de $4,0 \mu \mathrm{g} /$ $\mathrm{mL}$ (hipoadiponectinemia), se asocian en forma independiente con la presencia de enfermedades cardiovasculares, diabetes, obesidad y SM. En este trabajo hemos encontrado una prevalencia de $23,9 \%$ de hipoadiponectinemia en la población total. En la literatura se reportan resultados disímiles ${ }^{(9,17)}$, así en un grupo de coreanos no obesos la prevalencia es de $40,7 \%$ en hombres y $22,0 \%$ en mujeres; en un grupo de japoneses adultos la prevalencia es de $22,8 \%$ en hombres y $8,8 \%$ en mujeres mientras que en el presente 
trabajo se ha encontrado una prevalencia de $45,2 \%$ en hombres y 12,4\% en mujeres. Estas variaciones podrían deberse a diferencias genéticas o a factores ambientales como la dieta.

En el presente estudio se ha investigado la asociación de los niveles séricos de adiponectina con varios parámetros cardiometabólicos mediante el análisis de correlación. En lo que concierne a la edad, otros autores han encontrado ${ }^{(9,17)}$ que tiene asociación positiva con esta adipocina, y que la disminución en el aclaramiento de la adiponectina en el riñón puede ser la causa de los altos niveles de adiponectina que presentan las personas de mayor edad. En el presente trabajo no se ha observado relación entre edad de los sujetos y el nivel sérico de adiponectina.

La concentración sérica de adiponectina mostró una relación inversa con el IMC y la concentración de triglicéridos y relación directa con los niveles de HDL-col; esta relación se mantuvo después de ajustar el nivel de adiponectina por sexo, hechos que sugieren que la molécula estáasociadaa estos factores deriesgo cardiovascular independientemente del género. El presente trabajo concuerda con los resultados deotrosestudios ${ }^{(2,3)}$ y secreequeen los obesos las citoquinas (tales como Interleucina-6, y Factor de necrosis tumoral- $\alpha$,) están incrementadas y podrían causar disminución significativa de la expresión de ARN mensajero de la adiponectina y por tanto reducción en la secreción de la misma en los adipocitos $(2,15)$. Por otro lado, análisis in vitro e in vivo han demostrado que la adiponectina incrementa

los sistemas transportadores transmembrana de colesterol (ATP Binding Cassette Aı) y la síntesis de las apoliproteínas APO AI y APO AII) en el hígado (2), ambos factores son moléculas clave para el ensamblaje de las HDL, lo que explicaría la asociación positiva entre los niveles séricos de adiponectina y los de HDL-col; la adiponectina también interviene en el metabolismo de los triglicéridos, reduce a éstos en el plasma mediante el aumento del catabolismo de las VLDL-TG en el músculo esquelético a través de la elevación de la lipoprotein lipasa (LPL) en dicho tejido y de la expresión del receptor de VLDL ${ }^{(18)}$.

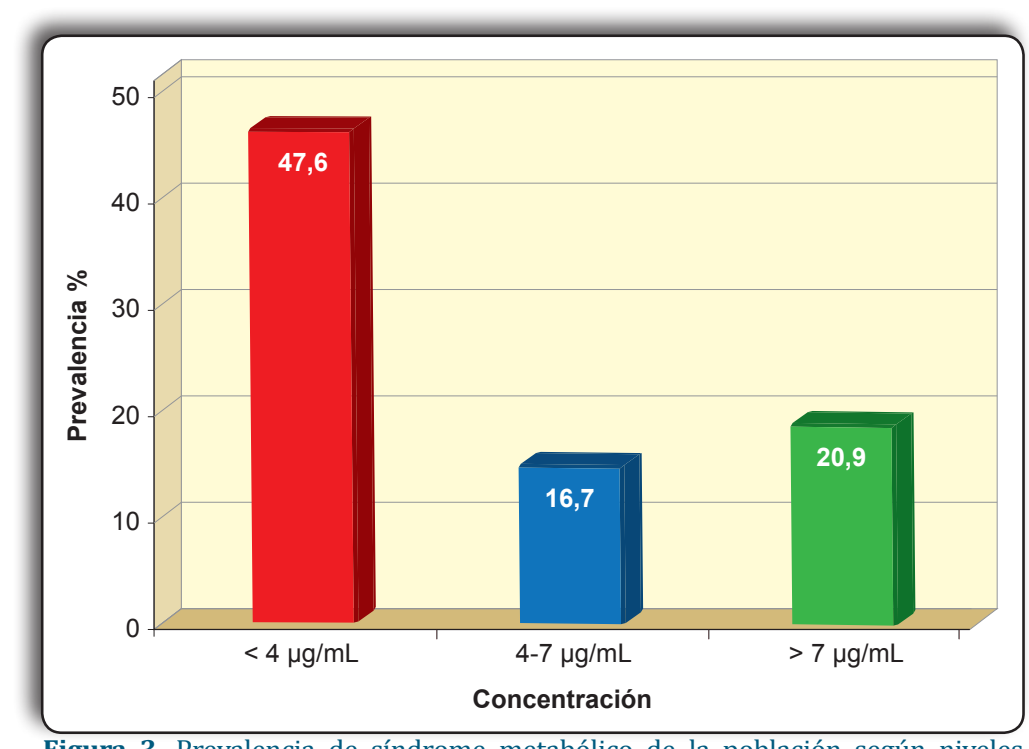

Figura 3. Prevalencia de síndrome metabólico de la población según niveles séricos de adiponectina, $p=0,037$.

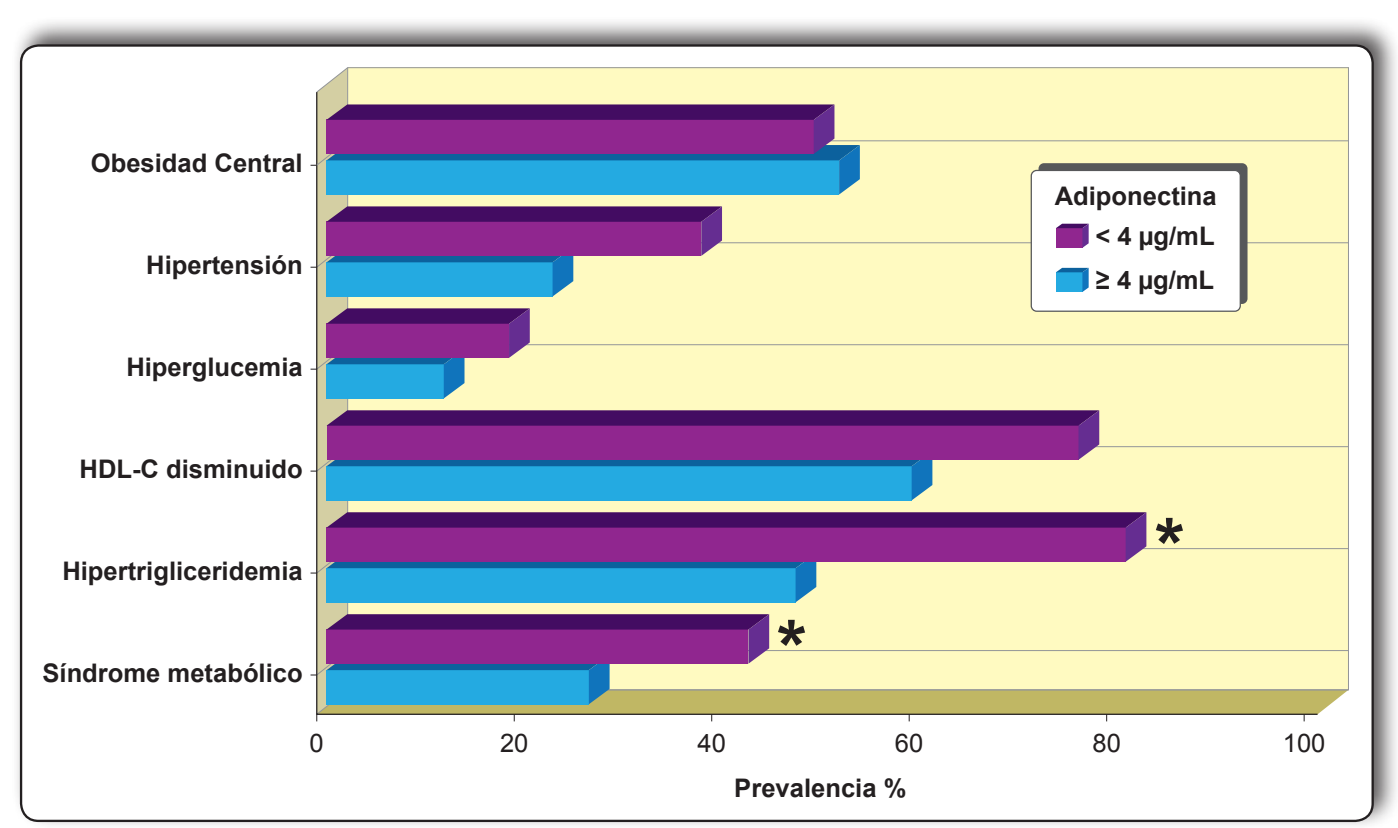

Figura 4. Prevalencia de los componentes de síndrome metabólico de la población según niveles séricos de adiponectina. ${ }^{*} p=<0,05$. 
En el presente estudio se ha investigado la relación entre los niveles de adiponectina sérica y la prevalencia de SM, así como la relación con sus cinco componentes definidos bajo criterio ATP III (11), incluyendo IMC.

Semuestraclaramentequelamayorprevalenciade SM $(47,6 \%)$ se encuentra en el grupo con adiponectina sérica $<4 \mu \mathrm{g} / \mathrm{mL}$ (hipoadiponectinemia); también se encontró que los niveles circulantes de adiponectina se asocian con algunos componentes individuales del SM y además con IMC, así, se encontró relación positiva significativa con HDL-col, negativa con IMC y triglicéridos, no se encontró asociación entre adiponectina y circunferencia de cintura, glucosa, y presión arterial, en estas dos últimas variables se debería a que en la altura normalmente los valores medios de glucosa sérica son menores y hay menor prevalencia de hipertensión que al nivel del mar, respectivamente. Los resultados también muestran que la prevalencia de los componentes del SM fue mayor (a excepción de la circunferencia de cintura) en el grupo con el nivel más bajo de adiponectina, aunque solamente el componente hipertrigliceridemia tuvo significación estadística; además, se observó que los niveles de adiponectina sérica disminuyen con la presencia de un número creciente de componentes del SM.

\section{CONCLUSIONES}

Los resultados obtenidos en el presente estudio permiten concluir que: las mujeres (a pesar de una mayor prevalencia de SM) presentan valores medios de adiponectina sérica más altos que los hombres. La relación significativa entre la adiponectina $\mathrm{y}$ algunos factores de riesgo cardiometabolico muestran claramente que la adiponectina sérica está fuertemente asociada con la prevalencia de SM y que en la poblaciones de altura la hipertrigliceridemia es el componente prevalente y se correlaciona negativamente con los niveles séricos de adiponectina.

\section{REFERENCIAS BIBLIOGRÁFICAS}

1. Scherer PE, Williams S, Fogliano M, Baldini G, Lodish HF. A novel serum protein similar to $C_{1}$, produced exclusively in adipocytes. J Biol Chem 1995; 270(45): 26746-9.

2. Iwaki M, Matsuda M, Maeda N, Funahashi T, Matsuzawa Y, Makishima M, et al. Induction of adiponectin, a fatderived antidiabetic and antiatherogenic factor, by nuclear receptors. Diabetes 2003; 52(7):1655-63.

3. Arita Y, Kihara S, Ouchi N, Takahashi M, Maeda K, Miyagawa J, et al. Paradoxical decrease of an adipose- specific protein, adiponectin, in obesity. Biochem Biophys Res Commun 1999; 257(1):79-83.

4. Weyer C, Funahashi T, Tanaka S, Hotta K, Matsuzawa Y, Pratley RE, et al. Hypoadiponectinemia in obesity and type 2 diabetes: close association with insulin resistance and hyperinsulinemia. J Clin Endocrinol Metab 2001; 86:1930-5.

5. Kadowaki T, Yamauchi T, Kubota N, Hara K, Ueki K, Tobe $\mathrm{K}$. Adiponectin and adiponectin receptors in insulin resistance, diabetes, and the metabolic syndrome. J Clin Invest 2006; 116(7):1784-92.

6. Baratta R, Amato S, Degano C, Farina MG, Patanè G, Vigneri $\mathrm{R}$, et al. Adiponectin relationship with lipid metabolism is independent of body fat mass: evidence from both cross-sectional and intervention studies. J Clin Endocrinol Metab. 2004; 89(6): 2665-71.

7. Kumada M, Kihara S, Sumitsuji S, Kawamoto $\mathrm{T}$, Matsumoto S, Ouchi $\mathrm{N}$, et al. Association of hypoadiponectinemia with coronary artery disease in men. Arterioscler Thromb Vasc Biol 2003; 23: 85-89.

8. Hopkins TA, Ouchi N, Shibata R, Walsh K. Adiponectin actions in the cardiovascular system. Cardiovasc Res 2007; 74(1): 11-8.

9. Pereira RI et al. Circulating adiponectin levels are lower in latino versus non-Latino white patients at risk for cardiovascular disease, independent of adiposity measures. BMC Endocr Disord 2011; 11: 13. [En línea] último acceso el o7 julio 2011. Disponible en: http://www. biomedcentral.com/1472-6823/11/13

10. Gonzáles GF, Tapia V. Asociación de los diferentes niveles de hipoxemia en la altura con el perfil lipídico y la glucemia en varones y mujeres a 4,100 $\mathrm{m}$ de altitud en los Andes Centrales del Perú. Endocrinol Nutr 2013; 6o(2): 79-86.

11. National Cholesterol Education Program (NCEP). Third Report of the National Cholesterol Education Program (NCEP) Expert panel on detection, evaluation, and treatment of high blood cholesterol in adults (adult treatment panel III) final report. Circulation 2002; 106(25): 3143-3421.

12. Pajuelo J, Sánchez-Abanto J, Torres H, Miranda M. Prevalencia del síndrome metabólico en pobladores peruanos por debajo de 1 ooo y por encima de los 3 ooo msnm. An Fac Med 2012; 73(2): 101-6.

13. Mohanna S, Baracco R. Seclén S. Lipid profile, waist circumference, and body mass index in a high altitude population. High Alt Med Biol 2006; 7(3): 245-55.

14. Nishizawa H, Shimomura I, Kishida K, Maeda N, Kuriyama $\mathrm{H}$, et al. Androgens decrease plasma adiponectin, an insulin-sensitizing adipocyte-derived protein. Diabetes 2002; 51(9): 2734-41.

15. Ong KK, Frystyk J, Flyvbjerg A, Petry CJ, Ness A, Dunger DB. Sex-discordant associations with adiponectin levels and lipid profi les in children. Diabetes 2006; 55(5): 1337-41.

16. Im JA, Kim SH, Lee JW, Shim JY, Lee HR, Lee DC. Association between hypoadiponectinemia and 
cardiovascular risk factors in nonobese healthy adults. Correspondencia:

Metabolism 2006; 55(11): 1546- 50.

17. Isobe T, Saitoh S, Takagi S, Takeuchi H, Chiba Y, et al. Influence of gender, age, and renal function on plasma adiponectin level: the Tanno and Sobetsu Study. Eur J Endocrinol 2005; 153(1): 91-8.

18. Qiao L, Zou C, Van der Westhuyzen DR, Shao J. Adiponectin reduces plasma triglyceride by increasing VLDL triglyceride catabolism. Diabetes 2008; 57(7):1824-33.
Nombre:
Elizabeth Carranza Alva
Dirección:
Instituto Nacional de Biología Andina Facultad de Medicina - UNMSM.
e-mail: Av. Alfonso Ugarte 88o. Lima 1, Perú. ecarranzalva@gmail.com 